\title{
RNA sequencing analyses reveal novel differentially expressed genes and pathways in pancreatic cancer
}

\author{
Yixiang Mao ${ }^{1,2,5}$, Jianjun Shen ${ }^{4}$, Yue Lư ${ }^{4}$ Kevin Lin ${ }^{4}$, Huamin Wang ${ }^{3}$, Yanan Li², $^{2}$ \\ Ping Chang ${ }^{2}$, Mary G. Walker ${ }^{4}$ and Donghui $\mathbf{L i}^{2}$ \\ ${ }^{1}$ Department of Oncology, The First Affiliated Hospital of Soochow University, Suzhou 215007, China \\ ${ }^{2}$ Department of Gastrointestinal Medical Oncology, The University of Texas MD Anderson Cancer Center, Houston, Texas \\ 77030, USA \\ ${ }^{3}$ Department of Pathology and Department of Translational Molecular Pathology, The University of Texas MD Anderson \\ Cancer Center, Houston, Texas 77030, USA \\ ${ }^{4}$ Department of Epigenetics and Molecular Carcinogenesis, The University of Texas MD Anderson Cancer Center, Smithville, \\ Texas 78957, USA \\ ${ }^{5}$ Department of Medical Oncology, Fudan University Shanghai Cancer Center, Shanghai 200032, China \\ Correspondence to: Donghui Li, email: dli@mdanderson.org \\ Yixiang Mao, email: maoyix@suda.edu.cn \\ Keywords: pancreatic cancer, RNA sequencing, transcriptome, pathway analysis \\ Received: June 03, $2016 \quad$ Accepted: February 27, $2017 \quad$ Published: March 22, 2017
}

Copyright: Mao et al. This is an open-access article distributed under the terms of the Creative Commons Attribution License 3.0 (CC BY 3.0), which permits unrestricted use, distribution, and reproduction in any medium, provided the original author and source are credited.

\section{ABSTRACT}

Gene expression microarrays have identified many tumor markers and therapeutic targets for pancreatic ductal adenocarcinoma (PDAC). However, microarray profilings have limited sensitivity and are prone to cross-hybridization between homologous DNA fragments. Here, we perform a transcriptome analysis of paired tumor and adjacent benign pancreatic tissues from 10 patients who underwent resection for PDAC. We identify a total of $\mathbf{2 7 3 6}$ differentially expressed genes (DEGs) with false discovery rate less than 0.05, including 1554 upregulated, 1182 downregulated, and 6 microRNAs (miR-614, miR-217, miR-27b, miR-4451, miR-3609, and miR-612). Overexpression of five DEGs, i.e. KRT16, HOXA10, CDX1, SI, and SERPINB5 in tumors is confirmed by RT-PCR in 20 additional tissues. Overexpression of KRT16 in PDAC is also verified on protein level. In addition, top canonical pathways such as granulocyte adhesion and diapedesis pathway have been identified. Our study represents a comprehensive characterization of the PDAC transcriptome and provides insight to the mechanisms of pancreatic carcinogenesis and potential biomarkers and novel therapeutic targets for pancreatic cancer.

\section{INTRODUCTION}

Pancreatic ductal adenocarcinoma (PDAC) is a highly lethal disease with a 5-year survival rate of $6 \%$ [1]. PDAC is usually diagnosed at late stage which prelude the chance of tumor resection for cure. PDAC is also highly aggressive and resistant to most therapies. Previous studies of largescale gene expression analysis have used the microarray approach to identify novel tumor markers and potential therapeutic targets for PDAC [2]. However, microarray analyses have limited sensitivity and are prone to crosshybridization between homologous DNA fragments [3].
With the advancement of the next-generation sequencing technologies, RNA sequencing (RNA-seq) has become a useful tool in defining the transcriptomes of cells. Compared to microarray analysis, RNA-seq has the advantage of higher sensitivity and the ability to detect splicing isoforms and somatic mutations [4, 5]. A few studies have been conducted in pancreatic cancer using RNA-seq method, but most of these studies were conducted in cell lines [6] and circulating tumor cells $[7,8]$. Gene expression profiling in PDAC tissue samples using the microarray approach were mostly conducted in patients with PDAC versus patients without cancer [9-12] or in tissue samples from PDAC patients 
with different clinical or pathological features [13-16]. Literature search failed to find any transcriptome analysis in comparing the tumor and adjacent benign pancreatic tissues in pancreatic cancer. To fill in this gap, we embarked on a study using RNA-seq to compare the transcriptomes of 10 paired tumor and adjacent benign pancreatic tissue samples from patients who underwent resection for PDAC. Novel differentially expressed genes and canonical pathways were identified by this approach, which may open new research venue for pancreatic cancer.

\section{RESULTS}

\section{RNA-seq}

RNA-seq was successfully carried out in all 20 samples. All sequence data were read at a length of $2 \times 76$ bp with high-quality metrics ( $>28$ Phred score) and nucleotide distributions. The total number of sequenced reads ranged from 25 million to 33 million pairs, and an average $95.5 \%$ (range: $92.2 \%-97.6 \%$ ) of the pairs were aligned to the hg19 genome assembly using the TopHat2 aligner. The percentage of genomic alignment was similar between the tumor and non-tumor tissues (mean \pm standard deviation: $96.1 \pm 1.1 \%$ and $95.0 \pm 1.8 \%$, respectively), suggesting no obvious detectable biases in the sequence data $(P=0.11)$. Alignment statistics indicated the data were of high quality and were uniform (i.e., no outliers with reference to alignment proficiency) and provided sufficient sequencing depth to pursue differential expression testing between two groups.

\section{Estimated purity of the tissue samples}

The purity of tumor and adjacent non-tumor tissue used in RNA-seq was $0.73 \pm 0.10$ and $0.80 \pm$ 0.08 , respectively as predicted by the "Estimation of STromal and Immune cells in MAlignant Tumours using Expression data" (ESTIMATE) method (paired t-test, $P=$ $0.10)$. There was no significant correlation between these two groups $(\mathrm{r}=0.124, P=0.73)$.

\section{Identification of DEGs}

We identified 2736 DEGs with false discovery rate $(\mathrm{FDR})<0.05$ including 1554 upregulated and 1182 downregulated genes (Supplementary Table 3). Although RNA-seq was trimmed to detect mRNA, we found that 6 microRNAs were enriched in the DEGs: two were upregulated (miR-614 and miR-612), and four were downregulated (miR-217, miR-27b, miR-4451, and miR3609) (Table 1). To select DEGs, we ranked genes by the $\log 10 P$ value of genes with FDR (q-value) $<0.05$ and plotted them against the $\log 2$ fold change in a "volcano" plot (Figure 1). We identified 17 genes that were upregulated and 36 genes that were downregulated with FDR (q-value) $<0.001$ and $\log$ ratio $\geq 5$ (Table 1 ). Among the 17 overexpressed genes, CDX1 (caudal type homeobox 1) had the highest fold difference in tumor versus non-tumor tissues followed by SI (sucrase-isomaltase, aka alpha-glucosidase), KRT16 (keratin 16) and SERPINB5 (serpin peptidase inhibitor, clade B (ovalbumin), member 5). SERPINB5 followed by KRT16 and HOXA10 had the smallest $P$ values and FDR q-values. The 36 downregulated genes included many genes coding for digestive enzymes, which reflect the impairments of exocrine pancreatic functions by the tumor.

\section{Validation analysis using quantitative RT-PCR and IHC}

Among the 17 upregulated genes, we selected the top five, i.e. $C D X 1, S I, K R T 16, H O X A 10$, and SERPINB5 for validation using RT-PCR in the 20 pairs of tumor and non-tumor tissues that were not used in RNAseq. The RT-PCR results confirmed overexpression of all five genes in pancreatic tumors compared to nontumor tissues (Figure 2). The largest fold difference in mRNA expression between tumor and non-tumor tissues was seen for SERPINB5 and KRT16. Because KRT16 protein expression has not been previously investigated in pancreatic cancer, we further conducted immunohistochemistry (IHC) to compare the expression level of KRT16 protein in eight pairs of tumor and adjacent non-tumor tissues from patients who underwent resection for PDAC. KRT16 staining was present in both cytoplasm and nucleus of the normal ductal epithelia (Figure 3, upper panels) and tumor cells (Figure 3, lower panel). But the protein expression was mostly detected in the cytoplasm. Tumor tissues showed a significantly higher level of KRT16 expression than non-tumor tissues, especially in cytoplasm. The average H-score for KRT16 expression was $236.1 \pm 46.8$ in tumors and $135.8 \pm 56.8$ in non-tumor tissues, respectively $(P=$ $0.002)$.

\section{IPA analyses of DEGs}

Ingenuity Pathway Analysis (IPA; Ingenuity Systems/Qiagen, Redwood City, CA, USA) of DEGs with a FDR q-value of $<0.01$ revealed 99 significant canonical pathways (Supplementary Table 4) and 21 significant molecular and cellular functions (Supplementary Table 5) (Fisher's exact test, $P<0.05)$. The top five canonical pathways are the granulocyte adhesion and diapedesis, inhibition of matrix metalloproteases, lipopolysaccharide/ interleukin-1-mediated inhibition of retinoid X receptor function, antigen presentation, and complement system pathways. The major contribution genes to each of the five pathways are listed in Table 2. The five top cellular functions that are over-represented by DEGs are cellular growth and proliferation, cellular movement, cell death and survival, cell to cell signaling and interactions, and cellular development (Supplementary Table 5). 
Table 1: Top differentially expressed genes $(F D R<0.001$ and $\log 2$ ratio $\geq 5)$ and miRNAs

\begin{tabular}{|c|c|c|c|c|}
\hline Symbol & Gene Name & Log2Ratio & $P$-value & FDR (q-value) \\
\hline \multicolumn{5}{|l|}{ Upregulated } \\
\hline$C D X 1$ & caudal type homeobox 1 & 8.166 & $2.28 \times 10^{-5}$ & $7.83 \times 10^{-4}$ \\
\hline$S I$ & sucrase-isomaltase (alpha-glucosidase) & 7.111 & $2.42 \times 10^{-8}$ & $8.73 \times 10^{-6}$ \\
\hline KRT16 & keratin 16 & 6.917 & $9.18 \times 10^{-13}$ & $7.94 \times 10^{-9}$ \\
\hline SERPINB5 & serpin peptidase inhibitor, clade B (ovalbumin), member 5 & 6.561 & $2.80 \times 10^{-14}$ & $4.85 \times 10^{-10}$ \\
\hline$T I N A G$ & tubulointerstitial nephritis antigen & 6.286 & $5.46 \times 10^{-8}$ & $1.43 \times 10^{-5}$ \\
\hline CST1 & cystatin SN & 6.250 & $2.27 \times 10^{-8}$ & $8.36 \times 10^{-6}$ \\
\hline PITX1 & paired-like homeodomain 1 & 6.081 & $2.78 \times 10^{-8}$ & $9.47 \times 10^{-6}$ \\
\hline HOXA10 & homeobox A10 & 6.054 & $2.70 \times 10^{-12}$ & $1.28 \times 10^{-8}$ \\
\hline LINC00460 & long intergenic non-protein coding RNA 460 & 5.987 & $7.85 \times 10^{-8}$ & $1.76 \times 10^{-5}$ \\
\hline$U G T 1 A 9$ & UDP glucuronosyltransferase 1 family, polypeptide A8 & 5.978 & $1.23 \times 10^{-10}$ & $1.78 \times 10^{-7}$ \\
\hline$S L C O 1 B 7$ & solute carrier organic anion transporter family, member 1B7 & 5.772 & $9.51 \times 10^{-7}$ & $9.84 \times 10^{-5}$ \\
\hline HOTTIP & HOXA distal transcript antisense RNA & 5.707 & $3.37 \times 10^{-6}$ & $2.19 \times 10^{-4}$ \\
\hline$S L C 6 A 14$ & solute carrier family 6 (amino acid transporter), member 14 & 5.586 & $2.96 \times 10^{-12}$ & $1.28 \times 10^{-8}$ \\
\hline$C E A C A M 5$ & carcinoembryonic antigen-related cell adhesion molecule 5 & 5.582 & $9.32 \times 10^{-9}$ & $4.61 \times 10^{-6}$ \\
\hline$M S L N$ & mesothelin & 5.187 & $5.31 \times 10^{-7}$ & $6.81 \times 10^{-5}$ \\
\hline TMPRSS4 & transmembrane protease, serine 4 & 5.118 & $1.80 \times 10^{-11}$ & $6.23 \times 10^{-8}$ \\
\hline$S L C O 1 B 3$ & solute carrier organic anion transporter family, member 1B3 & 5.031 & $1.47 \times 10^{-7}$ & $2.62 \times 10^{-5}$ \\
\hline \multicolumn{5}{|c|}{ Downregulated* } \\
\hline$S Y C N$ & syncollin & -8.059 & $1.70 \times 10^{-6}$ & $1.44 \times 10^{-4}$ \\
\hline$P L A 2 G 1 B$ & phospholipase A2, group IB (pancreas) & -7.822 & $2.01 \times 10^{-5}$ & $7.17 \times 10^{-4}$ \\
\hline$G P 2$ & glycoprotein 2 (zymogen granule membrane) & -7.728 & $1.63 \times 10^{-5}$ & $6.14 \times 10^{-4}$ \\
\hline RBPJL & $\begin{array}{l}\text { recombination signal binding protein for immunoglobulin kappa } \\
\qquad \mathrm{J} \text { region-like }\end{array}$ & -6.646 & $8.63 \times 10^{-7}$ & $9.24 \times 10^{-5}$ \\
\hline SERPINI2 & serpin peptidase inhibitor, clade I (pancpin), member 2 & -6.620 & $3.63 \times 10^{-7}$ & $5.18 \times 10^{-5}$ \\
\hline PRSS3 & protease, serine, 3 & -6.240 & $5.50 \times 10^{-6}$ & $3.05 \times 10^{-4}$ \\
\hline$K L K 1$ & kallikrein 1 & -6.224 & $2.31 \times 10^{-6}$ & $1.74 \times 10^{-4}$ \\
\hline$E R P 27$ & endoplasmic reticulum protein 27 & -6.103 & $1.51 \times 10^{-6}$ & $1.34 \times 10^{-4}$ \\
\hline$P D I A 2$ & protein disulfide isomerase family A, member 2 & -6.044 & $6.12 \times 10^{-7}$ & $7.51 \times 10^{-5}$ \\
\hline$A Q P 8($ Ins) & aquaporin 8 & -5.982 & $4.63 \times 10^{-6}$ & $2.76 \times 10^{-4}$ \\
\hline CLPSL1 & colipase-like 1 & -5.977 & $5.12 \times 10^{-9}$ & $2.95 \times 10^{-6}$ \\
\hline GPHA2 & glycoprotein hormone alpha 2 & -5.868 & $2.58 \times 10^{-6}$ & $1.86 \times 10^{-4}$ \\
\hline GUCA1C & guanylate cyclase activator $1 \mathrm{C}$ & -5.841 & $5.92 \times 10^{-7}$ & $7.42 \times 10^{-5}$ \\
\hline GSTA2 & glutathione S-transferase alpha 2 & -5.827 & $2.73 \times 10^{-6}$ & $1.91 \times 10^{-4}$ \\
\hline TMEM52 & transmembrane protein 52 & -5.711 & $1.38 \times 10^{-5}$ & $5.52 \times 10^{-4}$ \\
\hline PNLIPRP2 & pancreatic lipase-related protein 2 & -5.596 & $2.95 \times 10^{-5}$ & $9.39 \times 10^{-4}$ \\
\hline$A T P 4 A$ & ATPase, $\mathrm{H}+/ \mathrm{K}+$ exchanging, alpha polypeptide & -5.319 & $1.82 \times 10^{-9}$ & $1.44 \times 10^{-6}$ \\
\hline PM20D1 & peptidase M20 domain containing 1 & -5.251 & $2.34 \times 10^{-6}$ & $1.74 \times 10^{-4}$ \\
\hline C12orf39 & chromosome 12 open reading frame 39 & -5.045 & $4.83 \times 10^{-8}$ & $1.33 \times 10^{-5}$ \\
\hline$C C K B R$ (Ins) & cholecystokinin B receptor & -5.041 & $5.81 \times 10^{-8}$ & $1.44 \times 10^{-5}$ \\
\hline
\end{tabular}




\begin{tabular}{lcccc}
\hline Symbol & Gene Name & Log2Ratio & $P$-value & FDR (q-value) \\
\hline MiRNA & & & & \\
miR-614 & 3.64 & $3.36 \times 10^{-7}$ & $4.92 \times 10^{-5}$ \\
miR-217 & -4.21 & $2.64 \times 10^{-6}$ & $1.87 \times 10^{-4}$ \\
miR-27b & -2.15 & $3.10 \times 10^{-4}$ & 0.0048 \\
miR-4451 & -1.74 & $1.09 \times 10^{-3}$ & 0.012 \\
miR-3609 & -1.15 & $1.57 \times 10^{-3}$ & 0.015 \\
miR-612 & 0.91 & $4.81 \times 10^{-3}$ & 0.034 \\
\hline
\end{tabular}

*Sixteen downregulated genes that are coding for pancreatic digestive enzymes are not listed. The complete list of the downregulated genes are presented in Supplementary Table 3.

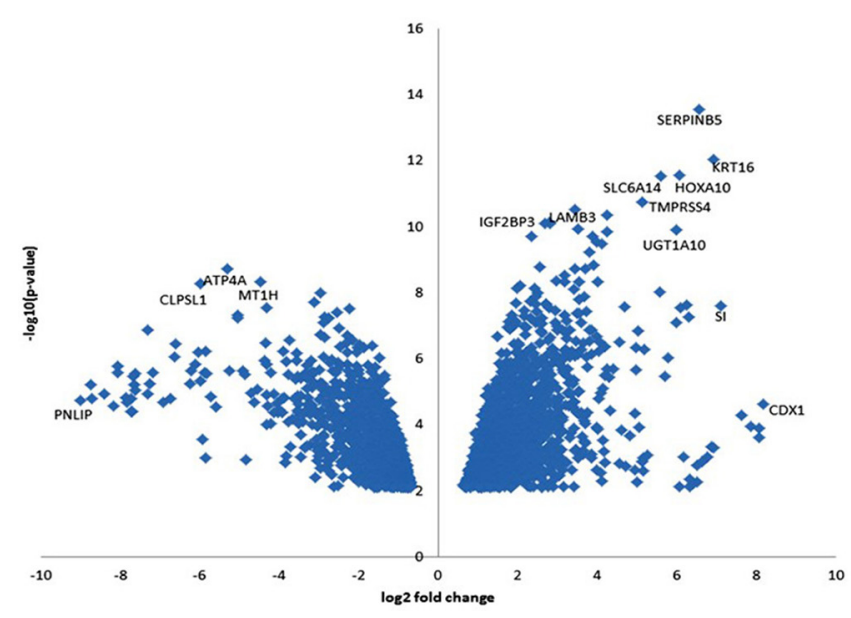

Figure 1: Volcano plot of DEGs $(P D R<0.05)$ in tumor and adjacent benign pancreatic tissues. The horizontal axis is the $\log 2$ fold change between PDAC and adjacent benign pancreatic tissues. The negative $\log 10$ of the $P$-value of Fisher's exact test is plotted on the vertical axis. Each gene is represented by one point on the graph.

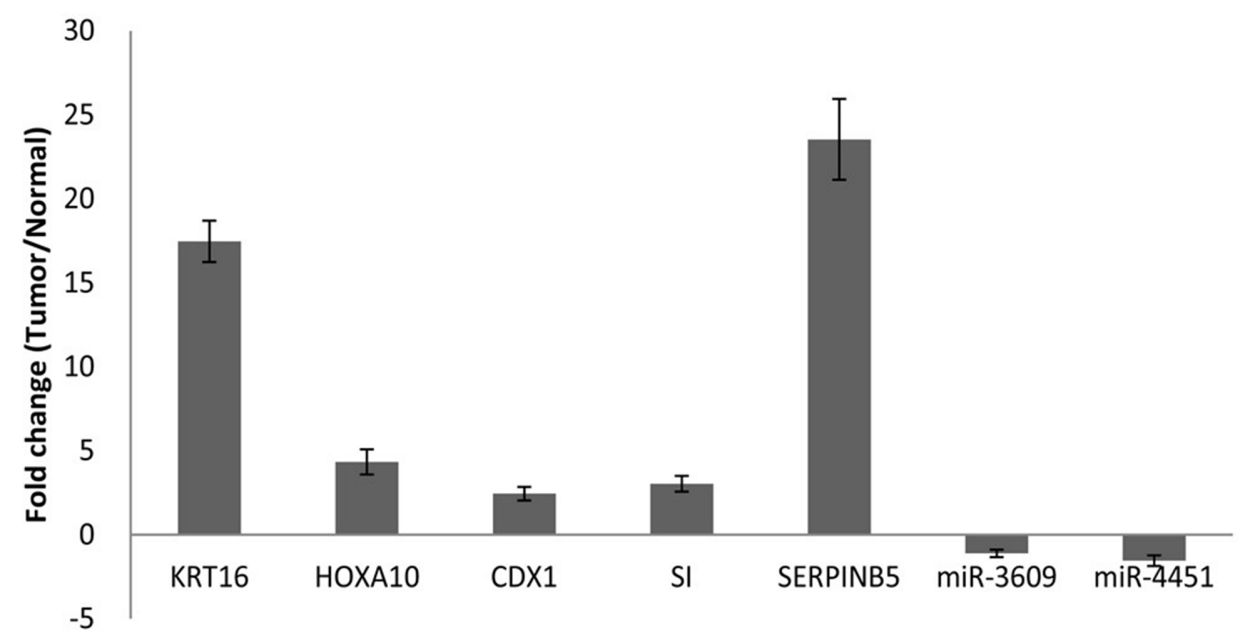

Figure 2: qRT-PCR analysis of KRT16, HOXA10, CDX1, SI, SERPINB5, miR-3609, and miR-4451 in PDAC. Real-time quantitative PCR was performed with gene-specific primers. The expression of each gene was normalized with the average expression of the endogenous reference gene $\beta$-actin. The logarithm of relative quantitation in the gene expression of corresponding transcripts in 20 tumor tissues compared to 20 adjacent non-tumor tissues is plotted in the graph. The error bar indicates the standard error of the mean fold change. 


\section{Upstream transcriptors enriched by DEGs}

The 15 most significantly activated or inhibited upstream transcription regulators identified by IPA are listed in Table 3. Among the inhibited upstream transcriptors are important tumor suppressor genes, such as TP53, CDKN2A and RB1. On the other hand, the activated upstream regulators mostly are signal transducers that play critical roles in inflammatory or immune response and tumorigenesis, e.g. STAT3, CTNNB1, SP1, and $N F \kappa B$ etc. Notably, two pancreatic cancer susceptibility genes previously identified by genome wide association studies, i.e. NR5A2 (nuclear receptor group 5A member 2) [17] and HNF1A (hepatocyte nuclear factor 1 homeobox A) [18], were among the inhibited upstream transcription regulators.

\section{DISCUSSION}

To our knowledge, this is the first report of a comprehensive transcriptome analysis using RNA-seq in pancreatic cancer. In 10 pairs of PDAC tumor and adjacent benign pancreatic tissues, a large number $(2,736)$ of DEGs were identified. Validation of overexpression of the top five DEGs at the RNA or protein levels suggest their potential values as biomarker or therapeutic targets in pancreatic cancer. IPA analysis has revealed several canonical pathways and molecular functions that are associated with pancreatic cancer. These findings opened new research venues for pancreatic cancer.

Using the RNA-seq technique, we identified much more DEGs in the current study compared with previous expression profiling analysis that used the microarray
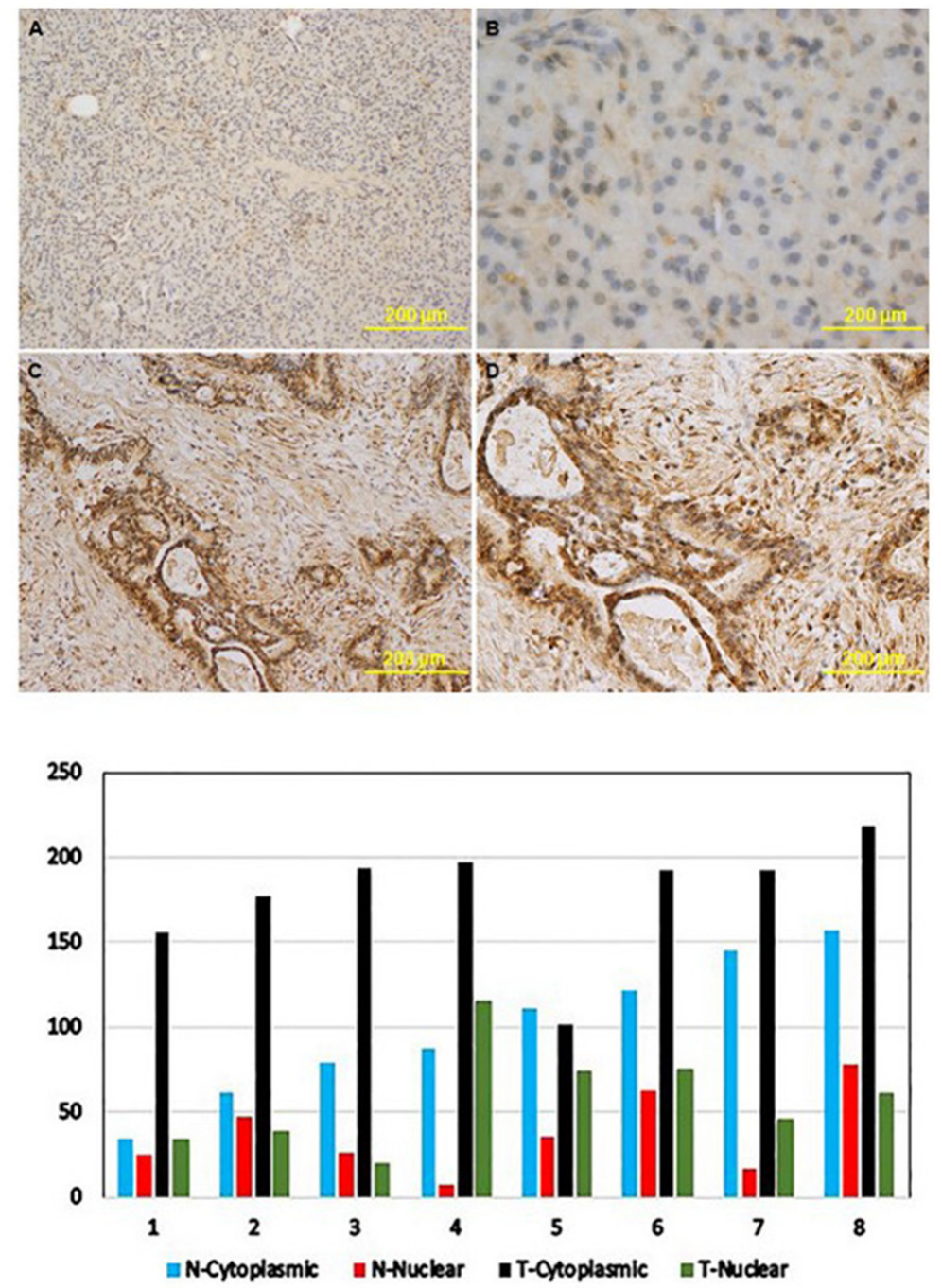

Figure 3: The expression levels of KRT16 protein in paired tumor and benign pancreatic tissues from patients who underwent resection for PDAC. Upper panels: immunohistochemistry images: (A) and (B), KRT16 expression in normal pancreatic tissues; (C) and (D), KRT16 expression in PDAC. Magnification was x40 for panel (A) and (C) and x100 for panel (B) and (D). Lower pane: KRT16 staining scores in PDAC (T) and benign pancreatic tissues $(\mathrm{N})$. 
Table 2: Top canonical pathways and molecular functions enriched by DEGs*

\begin{tabular}{|c|c|c|c|}
\hline $\begin{array}{l}\text { Canonical } \\
\text { pathways }\end{array}$ & $P$-value & Ratio & Molecules \\
\hline $\begin{array}{l}\text { Granulocyte } \\
\text { Adhesion and } \\
\text { Diapedesis }\end{array}$ & $8.56 \times 10^{-8}$ & $\begin{array}{l}33 / 177 \\
(0.186)\end{array}$ & $\begin{array}{c}\text { FPR3,IL1A,MMP3,MMP14,MMP13,CCL20,CCL22,CXCL5,IL1R2, } \\
\text { CXCL10,HRH1,CCL13,CXCL13,CCL28,MMP11,CXCL17,MMP12, } \\
\text { TNFRSF1B,MMP1,TNFRSF11B,CLDN10,SDC1,MMP28,ITGA2,MMP10, } \\
\text { ITGAL,SELPLG,C5,ITGB2,ITGAM,IL1RN,CCL18,MMP9 }\end{array}$ \\
\hline $\begin{array}{l}\text { Inhibition } \\
\text { of Matrix } \\
\text { Metalloproteases }\end{array}$ & $5.95 \times 10^{-6}$ & $\begin{array}{l}12 / 39 \\
(0.308)\end{array}$ & $\begin{array}{c}\text { SDC1,MMP28,MMP3,TIMP1,MMP14,MMP10,MMP13,MMP11,MMP12, } \\
\text { MMP9,LRP1,MMP1 }\end{array}$ \\
\hline $\begin{array}{l}\text { LPS/IL-1 Mediated } \\
\text { Inhibition of RXR } \\
\text { Function }\end{array}$ & $1.16 \times 10^{-5}$ & $\begin{array}{l}33 / 219 \\
(0.151)\end{array}$ & $\begin{array}{c}\text { IL1A,CHST4,CYP2C9,ABCG1,CYP2C19,IL1R2,ALDH1A1,UST,NR0B2, } \\
\text { NR112,ALDH3A2,ACSL5,HS6ST2,CHST11,HS3ST1,HS6ST3, } \\
\text { GSTA1,LBP,TNFRSF1B,SLCO1B3,ALDH6A1,TNFRSF11B,GSTA2, } \\
\text { GSTA4,IL4I1,TLR4,FABP2,SULT1E1,ALDH1L2,IL1RN,NR5A2, } \\
\text { GSTO2,SULT1B1 }\end{array}$ \\
\hline $\begin{array}{l}\text { Antigen } \\
\text { Presentation } \\
\text { Pathway }\end{array}$ & $2.11 \times 10^{-5}$ & $\begin{array}{l}11 / 37 \\
(0.297)\end{array}$ & $\begin{array}{c}P S M B 9, N L R C 5, H L A-A, H L A-D M B, C I I T A, H L A-D O B, P S M B 8, H L A- \\
F, T A P 1, T A P 2, T A P B P\end{array}$ \\
\hline $\begin{array}{l}\text { Complement } \\
\text { System }\end{array}$ & $2.11 \times 10^{-5}$ & $\begin{array}{c}11 / 37 \\
(0.297)\end{array}$ & ITGB2,CR1,ITGAM,C4BPB,CFB,C1QC,C6,C1QB,C2,CR2,C5 \\
\hline $\begin{array}{l}\text { Leukocyte } \\
\text { Extravasation } \\
\text { Signaling }\end{array}$ & $2.56 \times 10^{-5}$ & $\begin{array}{l}30 / 198 \\
(0.152)\end{array}$ & $\begin{array}{c}\text { RAC2,MMP3,PTK2B,MMP14,MMP13,RHOH,NOX1,TIMP1,CYBB, } \\
\text { MMP11,MMP12,MMP1,ACTN1,CLDN10,PIK3C2B,MMP28,ITGA2, } \\
\text { MMP10,ITGAL,SELPLG,ITGB2,WIPF1,ITGAM,EDIL3,RAP1GAP, } \\
\text { NCF2,PIK3R6,VAV1,MMP9,PRKCB }\end{array}$ \\
\hline
\end{tabular}

*Canonical pathway analysis identified the pathways from the IPA library of canonical pathways that were most significant enriched in differentially expressed genes. Genes with FDR q-value of $<0.01$ from data set that were associated with a canonical pathway in the Ingenuity Knowledge Base were considered for the analysis. The significance of the association between the data set and the pathway was measured in 2 ways: 1) a ratio of the number of molecules from the data set that map to the pathway divided by the total number of molecules that map to the canonical pathways is displayed. 2) Fisher's exact test was used to calculate a p-value determining the probability that the association between the genes in the observed values and the canonical pathway is explained by chance alone.

approach. RNA-seq is a more sensitive technology than expression profiling analysis using arrays, which is limited by its low sensitivity due to background hybridization and sometimes reduced specificity due to cross-hybridization of probes and targets [19, 20]. Comprehensive characterization of the transcriptome of PDAC is critical to understanding the disease at a system-wide level, as any missing data would create a biased view of this deadly disease.

Among the five top DEGs, overexpression of SERPINB5 [9, 11, 21-23], HOXA10 [12, 24] and KRT16 $[9,10,15]$ at the mRNA level has previously been reported in pancreatic cancer. SERPINB5 expression has been associated with clinical outcome of several types of human cancers [25-27]. HOXA10 is a DNA-binding transcription factor that may regulate gene expression, morphogenesis, and differentiation. Keratin 16 expression is regulated by epithelial growth factor [28] and it regulates innate immune functions [29]. A higher expression of KRT16 was observed in tumor than its adjacent normal pancreatic tissue in our study. Overexpression of KRT16 mRNA has been identified as a prognostic markers in triple negative breast cancer [30]. Findings from the RNA-seq, RTPCR and IHC experiments in the current study provide additional support for their potential role in pancreatic cancer. CDX1 has been shown to inhibit beta-catenin/Tcell factor transcriptional activity [31]. SI plays a critical role in the digestion of dietary carbohydrates including starch, sucrose and isomaltose [32]. Along with six other top DEGs, i.e. TINAG, LINC00460, UGT1A9, SLCO1B7, HOTTIP, and $A L C O 1 B 3$, their expression status could not be found in the Pancreatic Cancer Database [33]. Among the top 34 downregulated genes, SYCN, RBPJL, CLPSL1, GPHA2, GUCA1C GSTA2, TMEM52, ATP4A, $P M 20 D 1$, and $C 12$ orf39 have not previously been reported in pancreatic cancer. The role of these DEGs in pancreatic cancer needs further investigation.

IPA analyses indicated that the DEGs were mostly enriched in 21 significant molecular and cellular functions and 99 significant canonical pathways, which 
Table 3: Top 15 significant upstream transcription regulators

\begin{tabular}{|c|c|c|c|}
\hline Upstream Regulator & Log Ratio & Activation z-score ${ }^{\#}$ & $P$-value of overlap ${ }^{\&}$ \\
\hline \multicolumn{4}{|l|}{ Inhibition } \\
\hline TP53 & & -2.353 & $3.34 \times 10^{-32}$ \\
\hline NUPRI & -2.52 & -3.977 & $4.62 \times 10^{-13}$ \\
\hline$N K X 2-3$ & & -3.455 & $5.88 \times 10^{-12}$ \\
\hline HNF 1A & & -2.359 & $8.82 \times 10^{-12}$ \\
\hline$C D K N 2 A$ & 1.737 & -3.219 & $1.34 \times 10^{-11}$ \\
\hline estrogen receptor & & -2.299 & $9.57 \times 10^{-10}$ \\
\hline$R B 1$ & & -4.286 & $3.74 \times 10^{-08}$ \\
\hline$T C F 3$ & & -3.116 & $4.32 \times 10^{-08}$ \\
\hline TRIM24 & & -3.714 & $6.26 \times 10^{-07}$ \\
\hline BCL6 & & -2.313 & $1.60 \times 10^{-06}$ \\
\hline$N R 5 A 2$ & -2.876 & -2.7 & $7.26 \times 10^{-06}$ \\
\hline SATB1 & -0.814 & -2.003 & $1.09 \times 10^{-05}$ \\
\hline IRF4 & 2.115 & -2.41 & $4.24 \times 10^{-05}$ \\
\hline$R B L 1$ & & -3.124 & $1.72 \times 10^{-04}$ \\
\hline$S P D E F$ & & -2.887 & $2.39 \times 10^{-04}$ \\
\hline \multicolumn{4}{|l|}{ Activation } \\
\hline STAT3 & & 2.924 & $5.78 \times 10^{-19}$ \\
\hline CTNNB1 & & 3.359 & $1.99 \times 10^{-15}$ \\
\hline$S P 1$ & & 2.06 & $4.79 \times 10^{-13}$ \\
\hline$C E B P B$ & & 2.866 & $2.62 \times 10^{-12}$ \\
\hline NFkB (complex) & & 6.314 & $1.43 \times 10^{-11}$ \\
\hline$T B X 2$ & & 4.99 & $1.62 \times 10^{-11}$ \\
\hline$I R F 1$ & & 3.759 & $2.76 \times 10^{-11}$ \\
\hline$I R F 7$ & 1.504 & 5.591 & $2.88 \times 10^{-10}$ \\
\hline FOXM1 & 2.152 & 4.395 & $5.12 \times 10^{-10}$ \\
\hline STAT1 & & 4.576 & $2.05 \times 10^{-08}$ \\
\hline ETS1 & 0.863 & 2.575 & $5.06 \times 10^{-08}$ \\
\hline$J U N$ & & 2.045 & $5.57 \times 10^{-08}$ \\
\hline$F O X O 1$ & & 3.242 & $9.35 \times 10^{-08}$ \\
\hline$E 2 F 3$ & 1.137 & 2.394 & $1.16 \times 10^{-06}$ \\
\hline$M B D 2$ & & 2.549 & $5.41 \times 10^{-06}$ \\
\hline
\end{tabular}

\#Activation z-score is a statistical parameter that determines whether an upstream transcription regulator has significantly more "activated" predictions than "inhibited" predictions $(z>0)$ or vice versa $(z<0)$. Here, significance means that we reject the hypothesis that predictions are random with equal probability.

\&Overlap $\boldsymbol{P}$-value measures whether there is a statistically significant overlap between the dataset genes and the genes that are regulated by a transcriptional regulator. It is calculated using Fisher's Exact Test, and significance is generally attributed to $P$-values $<0.01$. Since the regulation direction ("activating" or "inhibiting") of an edge is not taken into account for the computation of overlap $P$-values the underlying network also includes findings without associated directional attribute, such as protein-DNA (promoter) binding. 
provides important clues for understanding the molecular mechanisms of PDAC pathogenesis. The overlapping networks of pathways were closely related to inflammatory and immune response, regulation of the cell cycle, and nicotine and neurotransmitter degradation. The major cellular functions of the DEGs represented include the cellular growth and proliferation, cellular movement, cell death and survival, cell to cell signaling and interactions, and cellular development. In contrast to a previous report on loss of expression of antigen-presenting molecules in human PDAC and PDAC cell lines [34], we observed upregulation of many antigen presentation-related genes in PDAC tissues. This discrepancy can be explained by the fact that the previous study compared PDAC tissue samples with the benign pancreatic samples from patients with benign pancreatic disease and downregulation of expression of antigen processing and antigen-presenting molecules reflected tumor evading immune recognition and destruction. The current study compared tumor with adjacent benign pancreatic tissues from the same PDAC patients. The upregulation of antigen presenting molecules reflect an inflammatory feature of the $\operatorname{PDAC}[35,36]$.

Interestingly, although the RNA-seq was trimmed to detect mRNA, we found that 6 microRNAs in the DEGs, i.e. miR-614 and miR-612 were upregulated, miR-217, miR-27b, miR-4451, and miR-3609 were downregulated in PDAC tissues compared with adjacent tissues. IPA showed that miR-3609 and miR-4451 were related to PIGG mRNA which is involved in the biosynthesis of glycosylphosphatidylinositol anchor. The role of these 2 downregulated microRNAs in PDAC remains to be studied.

Taken together, the results of our RNA-Seq analysis suggest that malignant transformation of pancreatic ductal cells involves the perturbation of multiple important cellular pathways, including cell growth-related pathways, metabolism-related processes, and immune-related and miRNA-regulated pathways.

Tissue cellularity is always a great challenge in PDAC research because PDAC consists of a higher percentage of stromal cells than other solid tumors. The infiltrating stromal and immune cells form the major fraction of normal cells in tumor tissue and may interfere with the tumor signal in molecular studies. In the current study, we restricted our tissue samples for RNA-seq to those with $>70 \%$ tumor cells in the tumor samples. We also used the ESTIMATE method [37] in which gene expression signatures are used to infer the fraction of stromal and immune cells in tumor samples. The average tumor purity prediction of 2,463 samples using ESTIMATE signatures was $0.61 \pm 0.20$. Although secondgeneration sequencing platforms facilitate the use of more heterogeneous samples, they may still underestimate the differential expression between cancer and normal tissues. Future work using microdissected tumor cells will help increase the accuracy of prediction.
In conclusion, this study was the first to use the RNA-Seq platform to comprehensively characterize the PDAC transcriptome. We identified a number of genes that were dysregulated in PDAC and may serve as targets for biomarker evaluation and therapeutic intervention. Follow-up analysis of modulator genes found in this study might be useful for acquiring a deeper understanding of pathological changes in PDAC and for developing prospective diagnostic and intervention strategies.

\section{EXPERIMENTAL PROCEDURES}

\section{Tissue samples}

Paired tumor and adjacent benign pancreatic tissues were obtained from 30 patients with PDAC who underwent tumor resection at the Fudan University Cancer Hospital in Shanghai, China, from May 2010 to February 2012. Information on patients' demographics, tumor location, histopathologic tumor type, histologic grade, tumor stage, lymph node metastasis, serum CA199 level, and performance status were collected from medical records. The characteristics of the 30 patients are summarized in Supplementary Table 1. No patients had received preoperative therapy. Informed consent was obtained from all patients, and the study was approved by the Institute Research Ethics Committee at Fudan University. Fresh samples of tumor and adjacent benign pancreas from each patient were harvested immediately after the surgery, washed with sterile normal saline, frozen in RNAlater (ThermoFisher Scientific, Grand Island, NY) in liquid nitrogen overnight and then transferred to a $-80^{\circ} \mathrm{C}$ freezer. Tumor and adjacent benign pancreatic tissues samples were confirmed via histopathologic examination by frozen sections. Briefly, the samples of tumor and adjacent benign pancreatic tissue were frozen in optimum cutting temperature compound for sectioning. A $5 \mu \mathrm{M}$ sections were prepared from each sample for hematoxylin and eosin staining. The cellularity of tumor sections was determined microscopically by a pathologist. Sections from 10 patients with a cellularity of greater than $70 \%$ and no necrosis were selected for RNA-seq. The remaining 20 samples with cellularity ranging from $15 \%$ to $65 \%$ were used for validation experiments.

\section{RNA-Seq}

Total RNA was isolated from frozen tissue blocks containing about 50-100 mg tissues using TRI Reagent (Molecular Research Center Inc., OH) following the manufacturer's instructions. The quality, quantity, and integrity of the total RNA were evaluated using a NanoDrop1000 spectrophotometer and Bioanalyzer 2100 (Agilent Technologies, CA). Samples with a RNA quality (RIN) score of $>7.0$ was used in RNA-seq. A mRNAfocused, barcoded library was generated using TruSeq 
RNA Sample Preparation Kits (Illumina, CA) with the ovation RNA-Seq System V2 (NuGEN Technologies, Inc., San Carlos, CA). The libraries were sequenced on an Illumina HiSeq 2000 instrument (San Diego, CA) with 2x76-base pair (bp) paired end protocol at the Science Park NGS Facility. Totally 20 libraries (paired tumor and adjacent benign tissues) from 10 patients with resected PDAC were sequenced, generating 25-33 million pairs of reads per sample. Each pair of reads represents a cDNA fragment from the library

The quality of the sequencing data was analyzed by the bioinformatics team associated with the Science Park NGS Facility using FastQC (http://www.bioinformatics. babraham.ac.uk/projects/fastqc/). The reads were mapped to human genome (hg19) by TopHat (version 2.0.4) [38] and Bowtie2 (version 2.0.0-beta7) [39]. 92.2-97.6\% fragments were mapped to human genome. The number of fragments in each known gene from RefSeq database [40] (downloaded from UCSC Genome Browser on March 6,2013 ) was enumerated using htseq-count from HTSeq package (version 0.5.3p9) (http://www-huber.embl.de/ users/anders/HTSeq/)

Genes with less than 10 fragments in all the samples were removed before differential expression analysis. The differential expression between conditions was statistically assessed by $\mathrm{R} /$ Bioconductor package edgeR (version 2.6.10) [41]. Paired design model was used as suggested in edgeR user's guide. Genes with FDR $\leq 0.05$ were called as differentially expressed.

\section{Tissue purity estimation}

Pancreatic cancer consists of a high percentage of stromal cells. The infiltrating stromal and immune cells form the major fraction of normal cells in tumor tissue. These cells play an important role in cancer biology but may also interfere in the analysis of tumor-specific signals. To measure the fraction of stromal and immune cells in tumor samples, we applied the ESTIMATE method [37] in our data analysis.

\section{Validation of selected DEGs}

Top DEGs were selected for validation according to the following criteria: 1 ) a $\log$ ratio of $\geq 5 ; 2$ ) an FDR q-value $<0.001$; and 3 ) potential biological significance in PDAC. The mRNA levels of the selected genes were measured by quantitative RT-PCR using an ABI PRISM 7900HT thermocycler (Applied Biosystems, CA). Specific primers used in these experiments are listed in Supplementary Table 2 . All reactions were run in triplicate. $\beta$-actin was used for the normalization of expression data, and the $2^{-\Delta \Delta \mathrm{Ct}}$ method was applied [42].

IHC for protein expression was performed on formalin-fixed paraffin-embedded sections of 8 pairs of tumor and adjacent benign pancreatic tissues from patients with resected PDAC. The tissue sections were obtained from the National Cancer Institute supported Human Tissue Network. IHC used the ABC (avidin-biotinperoxidase complex) method and the protein expression level was scored semi-quantitatively by multiply the staining intensity (0-3) with the percentage (0-100) of positive tumor cells (histo-score, H-score) [43].

\section{Pathway analysis}

IPA was used to map 1,460 DEGs with a FDR q-value of $<0.01$ to gene ontology groups and biological pathways using the Ingenuity Knowledge Base as the reference [44]. Fisher's exact test was used to calculate a probability value to indicate the association between each gene in the list and IPA-curated pathways and biological functions. A P-value less than 0.05 was considered statistically significant overrepresentation of genes in a canonical pathway or gene ontology group (e.g., molecular and cellular functions).

\section{ACKNOWLEDGMENTS}

We thank Markeda Wade in the Department of Scientific Publications of The University of Texas MD Anderson Cancer Center for editing the manuscript.

\section{CONFLICTS OF INTEREST}

The authors have no conflicts of interests to disclose.

\section{GRANT SUPPORT}

This work was in part supported by grants from National Natural Science Foundation of China (NSFC) 30901765 (Y.M.), China Scholarship Council (Y.M.), The Natural Science Foundation of the First Affiliated Hospital of Soochow University (Y.M.), CPRIT Core Facility Support Awards RP120348 (J.S.), and the Sheikh Ahmed Center for Pancreatic Cancer Research Funds (D.L.).

\section{REFERENCES}

1. American Cancer Society. Cancer Facts \& Figures 2014. Atlanta: American Cancer Society. 2014; 19.

2. Grützmann R, Boriss H, Ammerpohl O, Lüttges J, Kalthoff H, Schackert HK, Klöppel G, Saeger HD, Pilarsky C. Metaanalysis of microarray data on pancreatic cancer defines a set of commonly dysregulated genes. Oncogene. 2005; 24:5079-88.

3. Zhao S, Fung-Leung WP, Bittner A, Ngo K, Liu X. Comparison of RNA-Seq and microarray in transcriptome profiling of activated T cells. PLoS One. 2014; 9:e78644. 
4. Mardis ER. The impact of next-generation sequencing technology on genetics. Trends Genet. 2008; 24:133-41.

5. Wang Z, Gerstein M, Snyder M. RNA-Seq: a revolutionary tool for transcriptomics. Nat Rev Genet. 2009; 10:57-63.

6. Jia J, Parikh H, Xiao W, Hoskins JW, Pflicke H, Liu X, Collins I, Zhou W, Wang Z, Powell J, Thorgeirsson SS, Rudloff U, Petersen GM, Amundadottir LT. An integrated transcriptome and epigenome analysis identifies a novel candidate gene for pancreatic cancer. BMC Med Genomics. 2013; 6:33.

7. Yu M, Ting DT, Stott SL, Wittner BS, Ozsolak F, Paul S, Ciciliano JC, Smas ME, Winokur D, Gilman AJ, Ulman MJ, Xega K, Contino G, et al. RNA sequencing of pancreatic circulating tumour cells implicates WNT signalling in metastasis. Nature. 2012; 487:510-13.

8. Ting DT, Wittner BS, Ligorio M, Vincent Jordan N, Shah AM, Miyamoto DT, Aceto N, Bersani F, Brannigan BW, Xega K, Ciciliano JC, Zhu H, MacKenzie OC, et al. Singlecell RNA sequencing identifies extracellular matrix gene expression by pancreatic circulating tumor cells. Cell Reports. 2014; 8:1905-18.

9. Logsdon CD, Simeone DM, Binkley C, Arumugam T, Greenson JK, Giordano TJ, Misek DE, Kuick R, Hanash $\mathrm{S}$. Molecular profiling of pancreatic adenocarcinoma and chronic pancreatitis identifies multiple genes differentially regulated in pancreatic cancer. Cancer Res. 2003; 63:2649-57.

10. Friess H, Ding J, Kleeff J, Fenkell L, Rosinski JA, Guweidhi A, Reidhaar-Olson JF, Korc M, Hammer J, Büchler MW. Microarray-based identification of differentially expressed growth- and metastasis-associated genes in pancreatic cancer. Cell Mol Life Sci. 2003; 60:1180-99.

11. Pfeffer F, Koczan D, Adam U, Benz S, von Dobschuetz E, Prall F, Nizze H, Thiesen HJ, Hopt UT, Löbler M. Expression of connexin26 in islets of Langerhans is associated with impaired glucose tolerance in patients with pancreatic adenocarcinoma. Pancreas. 2004; 29:284-90.

12. Iacobuzio-Donahue CA, Ashfaq R, Maitra A, Adsay NV, Shen-Ong GL, Berg K, Hollingsworth MA, Cameron JL, Yeo CJ, Kern SE, Goggins M, Hruban RH. Highly expressed genes in pancreatic ductal adenocarcinomas: a comprehensive characterization and comparison of the transcription profiles obtained from three major technologies. Cancer Res. 2003; 63:8614-22.

13. Kim HN, Choi DW, Lee KT, Lee JK, Heo JS, Choi SH, Paik SW, Rhee JC, Lowe AW. Gene expression profiling in lymph node-positive and lymph node-negative pancreatic cancer. Pancreas. 2007; 34:325-34.

14. Campagna D, Cope L, Lakkur SS, Henderson C, Laheru D, Iacobuzio-Donahue CA. Gene expression profiles associated with advanced pancreatic cancer. Int J Clin Exp Pathol. 2008; 1:32-43.

15. Buchholz M, Braun M, Heidenblut A, Kestler HA, Klöppel G, Schmiegel W, Hahn SA, Lüttges J, Gress
TM. Transcriptome analysis of microdissected pancreatic intraepithelial neoplastic lesions. Oncogene. 2005; 24:6626-36.

16. Chaika NV, Yu F, Purohit V, Mehla K, Lazenby AJ, DiMaio D, Anderson JM, Yeh JJ, Johnson KR, Hollingsworth MA, Singh PK. Differential expression of metabolic genes in tumor and stromal components of primary and metastatic loci in pancreatic adenocarcinoma. PLoS One. 2012; 7:e32996.

17. Petersen GM, Amundadottir L, Fuchs CS, Kraft P, Stolzenberg-Solomon RZ, Jacobs KB, Arslan AA, Buenode-Mesquita HB, Gallinger S, Gross M, Helzlsouer K, Holly EA, Jacobs EJ, et al. A genome-wide association study identifies pancreatic cancer susceptibility loci on chromosomes 13q22.1, 1q32.1 and 5p15.33. Nat Genet. 2010; 42:224-28.

18. Pierce BL, Ahsan H. Genome-wide "pleiotropy scan" identifies HNF1A region as a novel pancreatic cancer susceptibility locus. Cancer Res. 2011; 71:4352-58.

19. Pawitan Y, Michiels S, Koscielny S, Gusnanto A, Ploner A. False discovery rate, sensitivity and sample size for microarray studies. Bioinformatics. 2005; 21:3017-24.

20. Marioni JC, Mason CE, Mane SM, Stephens M, Gilad Y. RNA-seq: an assessment of technical reproducibility and comparison with gene expression arrays. Genome Res. 2008; 18:1509-17.

21. Mardin WA, Petrov KO, Enns A, Senninger N, Haier J, Mees ST. SERPINB5 and AKAP12 - expression and promoter methylation of metastasis suppressor genes in pancreatic ductal adenocarcinoma. BMC Cancer. 2010; 10:549.

22. Crnogorac-Jurcevic T, Missiaglia E, Blaveri E, Gangeswaran R, Jones M, Terris B, Costello E, Neoptolemos JP, Lemoine NR. Molecular alterations in pancreatic carcinoma: expression profiling shows that dysregulated expression of S100 genes is highly prevalent. J Pathol. 2003; 201:63-74.

23. Sato N, Maehara N, Goggins M. Gene expression profiling of tumor-stromal interactions between pancreatic cancer cells and stromal fibroblasts. Cancer Res. 2004; 64:6950-56.

24. Cui XP, Qin CK, Zhang ZH, Su ZX, Liu X, Wang SK, Tian XS. HOXA10 promotes cell invasion and MMP-3 expression via TGFß2-mediated activation of the p38 MAPK pathway in pancreatic cancer cells. Dig Dis Sci. 2014; 59:1442-51.

25. Takagi Y, Matsuoka Y, Shiomi T, Nosaka K, Takeda C, Haruki T, Araki K, Taniguchi Y, Nakamura H, Umekita Y. Cytoplasmic maspin expression is a predictor of poor prognosis in patients with lung adenocarcinoma measuring $<3 \mathrm{~cm}$. Histopathology. 2015; 66:732-39.

26. Ma S, Pang C, Song L, Guo F, Sun H. The expression of ATF3, MMP-2 and maspin in tissue chip of glioma. Pak J Pharm Sci. 2015; 28:1059-63. 
27. Lionello M, Blandamura S, Staffieri C, Tealdo G, Giacomelli L, Marchese Ragona R, de Filippis C, Staffieri A, Marioni G. Postoperative radiotherapy for laryngeal carcinoma: the prognostic role of subcellular Maspin expression. Am J Otolaryngol. 2015; 36:184-89.

28. Chen YJ, Wang YN, Chang WC. ERK2-mediated C-terminal serine phosphorylation of p300 is vital to the regulation of epidermal growth factor-induced keratin 16 gene expression. J Biol Chem. 2007; 282:27215-28.

29. Lessard JC, Piña-Paz S, Rotty JD, Hickerson RP, Kaspar RL, Balmain A, Coulombe PA. Keratin 16 regulates innate immunity in response to epidermal barrier breach. Proc Natl Acad Sci USA. 2013; 110:19537-42.

30. Yu KD, Zhu R, Zhan M, Rodriguez AA, Yang W, Wong S, Makris A, Lehmann BD, Chen X, Mayer I, Pietenpol JA, Shao ZM, Symmans WF, Chang JC. Identification of prognosis-relevant subgroups in patients with chemoresistant triple-negative breast cancer. Clinical cancer research. 2013; 19:2723-2733. doi: 10.1158/1078-0432. CCR-12-2986.

31. Guo RJ, Huang E, Ezaki T, Patel N, Sinclair K, Wu J, Klein P, Suh ER, Lynch JP. Cdx1 inhibits human colon cancer cell proliferation by reducing beta-catenin/T-cell factor transcriptional activity. J Biol Chem. 2004; 279:36865-75.

32. Lin AH, Hamaker BR, Nichols BL Jr. Direct starch digestion by sucrase-isomaltase and maltase-glucoamylase. J Pediatr Gastroenterol Nutr. 2012; 55:S43-45.

33. Thomas JK, Kim MS, Balakrishnan L, Nanjappa V, Raju R, Marimuthu A, Radhakrishnan A, Muthusamy B, Khan AA, Sakamuri S, Tankala SG, Singal M, Nair B, et al. Pancreatic Cancer Database: an integrative resource for pancreatic cancer. Cancer Biol Ther. 2014; 15:963-67.

34. Pandha H, Rigg A, John J, Lemoine N. Loss of expression of antigen-presenting molecules in human pancreatic cancer and pancreatic cancer cell lines. Clin Exp Immunol. 2007; 148:127-35.

35. Hamada S, Masamune A, Shimosegawa T. Inflammation and pancreatic cancer: disease promoter and new therapeutic target. J Gastroenterol. 2014; 49:605-17.

36. Komura T, Sakai Y, Harada K, Kawaguchi K, Takabatake H, Kitagawa H, Wada T, Honda M, Ohta T, Nakanuma
Y, Kaneko S. Inflammatory features of pancreatic cancer highlighted by monocytes/macrophages and CD4+ T cells with clinical impact. Cancer Sci. 2015; 106:672-86.

37. Yoshihara K, Shahmoradgoli M, Martínez E, Vegesna R, Kim H, Torres-Garcia W, Treviño V, Shen H, Laird PW, Levine DA, Carter SL, Getz G, Stemke-Hale K, et al. Inferring tumour purity and stromal and immune cell admixture from expression data. Nat Commun. 2013; 4:2612.

38. Kim D, Pertea G, Trapnell C, Pimentel H, Kelley R, Salzberg SL. TopHat2: accurate alignment of transcriptomes in the presence of insertions, deletions and gene fusions. Genome Biol. 2013; 14:R36.

39. Langmead B, Salzberg SL. Fast gapped-read alignment with Bowtie 2. Nat Methods. 2012; 9:357-59.

40. Pruitt KD, Brown GR, Hiatt SM, Thibaud-Nissen F, Astashyn A, Ermolaeva O, Farrell CM, Hart J, Landrum MJ, McGarvey KM, Murphy MR, O’Leary NA, Pujar S, et al. RefSeq: an update on mammalian reference sequences. Nucleic Acids Res. 2014; 42:D756-63.

41. Robinson MD, McCarthy DJ, Smyth GK. edgeR: a Bioconductor package for differential expression analysis of digital gene expression data. Bioinformatics. 2010; 26:139-40.

42. Guescini M, Sisti D, Rocchi MB, Stocchi L, Stocchi V. A new real-time PCR method to overcome significant quantitative inaccuracy due to slight amplification inhibition. BMC Bioinformatics. 2008; 9:326.

43. Budwit-Novotny DA, McCarty KS, Cox EB, Soper JT, Mutch DG, Creasman WT, Flowers JL, McCarty KS Jr. Immunohistochemical analyses of estrogen receptor in endometrial adenocarcinoma using a monoclonal antibody. Cancer Res. 1986; 46:5419-25.

44. Calvano SE, Xiao W, Richards DR, Felciano RM, Baker HV, Cho RJ, Chen RO, Brownstein BH, Cobb JP, Tschoeke SK, Miller-Graziano C, Moldawer LL, Mindrinos $\mathrm{MN}$, et al. A network-based analysis of systemic inflammation in humans. Nature. 2005; 437:1032-37. 